\title{
Adaptive Phase Shaping in a Fiber Chirped Pulse Amplification System
}

\author{
Nikita K. Daga, Fei He, Hazel S.S. Hung, N. Naz, Jerry Prawiharjo, David C. Hanna, \\ David J. Richardson, David P. Shepherd \\ Optoelectronics Research Centre, University of Southampton, Southampton SO17 1BJ, UK \\ E-mail: nid@orc.soton.ac.uk
}

Abstract. We demonstrate adaptive spectral phase shaping in a fiber chirped pulse amplification system. The adaptive process, controlled by a simulated annealing algorithm, resulted in three times improvement in the autocorrelation peak intensity of $65 \mu \mathrm{J}$ pulses.

\section{Introduction}

Fiber lasers offer high efficiency and high beam quality, while also dissipating heat efficiently. In order to achieve high-power femtosecond pulses in fiber laser systems, chirped pulse amplification (CPA) is employed. Power scaling of the fiber CPA system is, however, non-trivial. The tight confinement of the fiber geometry leads to nonlinear effects, especially self-phase-modulation (SPM), while gain shaping in the fiber amplifiers limits the spectral bandwidth and alters the spectral profile. The long interaction length of the fiber introduces significant dispersion, which can not always be fully compensated by the compressor. The combined effect of these factors results in significant degradation of the pulse. In order to improve the pulse quality, a programmable pulse shaper can be implemented.

The use of phase modulators to correct for SPM-induced phase distortions up to $2 \pi$ has been demonstrated in fiber CPA systems, [1], and adaptive pulse shaping has been used to control the propagation of ultrashort pulses in optical fibers [2]. Recent work combining adaptive pulse shaping with fiber-based CPAs has been demonstrated with amplitude-only shaping [3]. This technique can be used to offset the gain shaping and SPM phase contribution, but cannot compensate for the residual dispersion in the system.

In this paper we demonstrate the use of spectral phase shaping in an adaptive fiber CPA system. The adaptive process was controlled by a simulated annealing algorithm and was used to optimize the coefficients of a Taylor expansion of the spectral phase profile. Three times improvement in the autocorrelation peak intensity was demonstrated, with close to transform-limited pulse durations of $800 \mathrm{fs}$ at pulse energies of $65 \mu \mathrm{J}$.

\section{Experimental Details}

A schematic of the CPA system is shown in Fig 1. The pump source is a mode-locked $1.053 \mu \mathrm{m} \mathrm{Nd}$ :glass laser delivering $600 \mathrm{fs}$ pulses at a repetition rate of $80 \mathrm{MHz}$. These pulses are shaped with a phase-only pulse shaper before being stretched to $\sim 1$ ns with a grating stretcher. An electro-optic modulator then reduces the repetition rate to $100 \mathrm{kHz}$ before amplification to $0.1 \mathrm{~W}$ using the core-pumped Yb-fiber pre-amplifiers. High pulse energies were achieved with two cladding-pumped amplifiers, each of which used a $2 \mathrm{~m}$ length of double-clad Yb-doped PCF from Crystal Fiber (core diameter $40 \mu \mathrm{m}$, NA 0.03 ; inner cladding diameter $170 \mu \mathrm{m}$, NA 0.6 ) and fiberised 
$975 \mathrm{~nm}$ pump laser diodes (diode powers were $6 \mathrm{~W}$ and $20 \mathrm{~W}$ respectively). Acoustooptic modulators prevented ASE build-up, and reduced the final repetition rate to $16.67 \mathrm{kHz}$. A dielectric grating compressor, with $65 \%$ overall transmission efficiency, recompressed the pulses. The maximum-to-minimum contrast of the autocorrelation trace within the measuring window (30ps) was used as the feedback parameter for the adaptive loop, which modified the phase profile applied at the shaper.

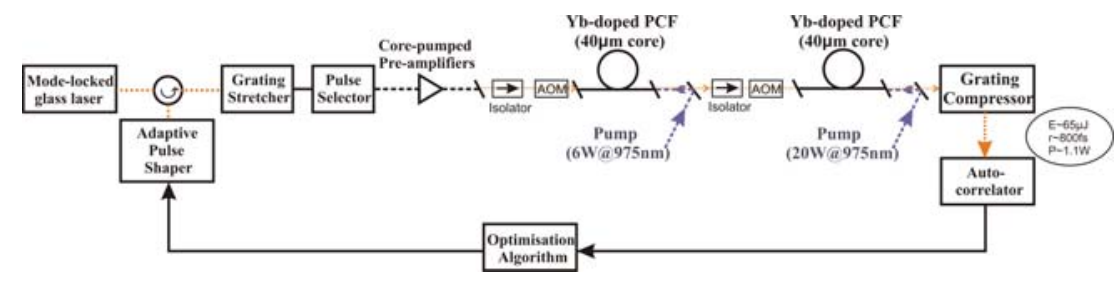

Fig. 1. Schematic of the CPA system

The pulse shaper is arranged in a 4-f configuration [4]. It consists of a diffraction grating with a groove density of 1100 lines $/ \mathrm{mm}$, cylindrical lens $(\mathrm{f}=200 \mathrm{~mm})$, spatial light modulator (SLM) and a flat mirror to retro-reflect the input beam. The SLM is a phase-only liquid crystal array of 128 pixels covering a distance of $12.8 \mathrm{~mm}$ (CRI SLM-128-MIR). The 10nm bandwidth input beam was expanded to a collimated beam radius of $5.9 \mathrm{~mm}$ and was incident upon the diffraction grating at an angle of 10 degrees, with a corresponding diffracted beam angle of 80 degrees. The spectral resolution as defined in ref. [4] was calculated to be $0.04 \mathrm{~nm}$. The maximum complexity, $\eta$, is limited by the number of pixels to 128 such that the finest achievable spectral feature, $\delta \lambda$, is $0.078 \mathrm{~nm}$ and the time window, T, is $20 \mathrm{ps}$.

In this work, we use an optimisation algorithm known as the Generalised Simulated Annealing (GSA) algorithm [5]. The main parameters that affect the convergence are the initial starting temperature, $\mathrm{T}_{0}$, the acceptance parameter, $\mathrm{q}_{\mathrm{a}}$, and the visiting parameter, $\mathrm{q}_{\mathrm{v}}$. Typically, the algorithm ran for 150 iterations with $\mathrm{T}_{0}$ set to be twice the expected value of the optimized feedback parameter (i.e. twice the maximum minus the minimum voltage of the autocorrelation), $\mathrm{q}_{\mathrm{a}}=1$, and $\mathrm{q}_{\mathrm{v}}=2.5$.

The phase was described by a Taylor series expansion so that the 128 pixels could all be addressed with only a few optimisation parameters and thus achieve fast convergence. The adaptive CPA system modified the $\beta_{2}$ to $\beta_{6}$ dispersion terms of the spectral phase. Figure 2 below shows a set of typical convergence data for the feedback parameter and the first three optimisation parameters. It can be seen from this data that the algorithm converges quickly within the first 100 iterations, which took $\sim 2$ minutes.
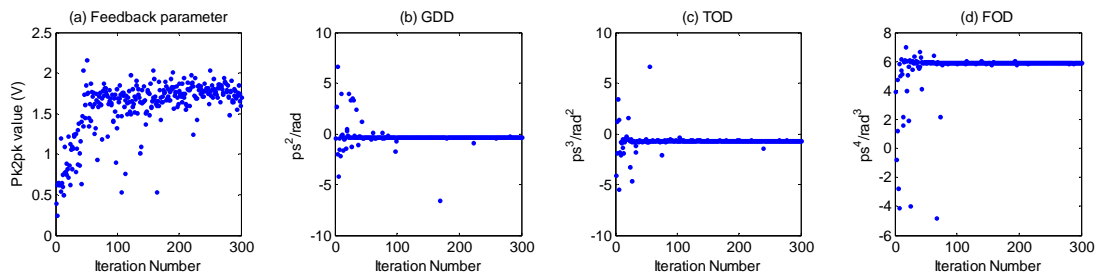
Fig. 2. Convergence data for (a) the feedback parameter and the (b) second, (c) third, and (d) fourth order dispersion parameters of the GSA algorithm.

To confirm the calibration of the pulse shaper, low energy pulses suffering from minimal SPM were adaptively compressed at various grating compressor roof mirror positions. The second order phase parameters obtained from these experiments agreed well with the expected change in dispersion from the compressor.

\section{Results}

The quality of the pulses without shaping is shown in Fig.3(a). The CPA system yields high quality pulses at low energy but a large pedestal remains at high pulse energies of $65 \mu \mathrm{J}$ which have a calculated B-integral of $\sim 2.5 \pi$. With the shaper in the system, Fig.3(b) shows that at $65 \mu \mathrm{J}$, the pulse quality is significantly improved with the pedestal almost completely depleted and with a three times improvement in the autocorrelation peak. The autocorrelation is compressed from $1.4 \mathrm{ps}$ to $1.1 \mathrm{ps}$, close to the flat phase limit calculated from the Fourier transform of the measured spectral data. The spectra after the shaper with and without shaping are shown in Fig.3(c) showing minimal spectral distortion due to the applied phase.

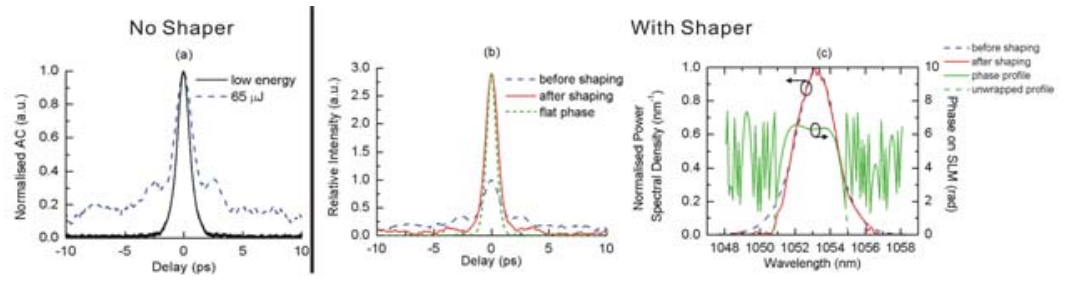

Fig. 3. Autocorrelations for (a) the unshaped pulses of the low energy and $65 \mu \mathrm{J}$ energy pulses and (b) the high energy shaped pulses. (c) The spectra after the shaper with and without shaping, and with the applied phase profile are also shown.

\section{Conclusions}

We have demonstrated high quality $65 \mu \mathrm{J}$ pulses from an adaptive fiber CPA system by controlling the spectral phase pre-compensating for SPM effects induced in the amplifier stages. A GSA algorithm was used to optimize the second to sixth order dispersion parameters of the phase resulting in pulse compression near to the flat phase limit within 150 iterations. A three-fold increase in the autocorrelation peak was also observed.

Further work into phase and amplitude shaping in fiber CPA systems, investigating the compensation of SPM at even higher energies in addition to alternative optimisation techniques, is anticipated.

1 F.G.Omenetto, D.H.Reitze, B.P.Luce, M.D.Moores, and A.J.Taylor, IEEE J. Select. Top. Quantum Electron. 8, 690, 2002.

2 G. Zhu, J. Edinberg, and C. Xu, Opt. Express 15, 2530-2534, 2007.

3 D. N. Schimpf, J. Limpert, and A. Tünnermann, , Opt. Express 15, 16945-16953, 2007.

4 A.M.Weiner, Rev. Sci. Instrum. 71, 1929, 2000. 
5 J Tsallis, C. and D. A. Stariolo, Physica A 233, (1-2): 395-406, 1996. 